\title{
PANORAMA DA HANSENÍASE ENTRE AS MACRORREGIÕES BRASILEIRAS DE 2015 A 2018
}

\section{ARTIGO ORIGINAL}

AZEVEDO, Vanessa Moita ${ }^{1}$

LACERDA, Bruna Soares ${ }^{2}$

FECURY, Amanda Alves ${ }^{3}$

AZEVEDO, Vanessa Moita. LACERDA, Bruna Soares. FECURY, Amanda Alves. Panorama da hanseníase entre as macrorregiões brasileiras de 2015 a 2018. Revista Científica Multidisciplinar Núcleo do Conhecimento. Ano 05, Ed. 03, Vol. 01, pp. 111-123. Março de 2020. ISSN: 2448-0959

\section{RESUMO}

Introdução: Hanseníase é uma doença de elevado risco de contágio. No Brasil, o número de casos notificados ainda é alto e varia entre as regiões. Conhecer o perfil epidemiológico da doença faz-se necessário para avaliar a efetividade das políticas de erradicação. Objetivo: Avaliar o panorama epidemiológico da Hanseníase entre as macrorregiões brasileiras, no período de 2015 a 2018. Métodos: Realizou-se um estudo analítico, retrospectivo, transversal, por meio do levantamento de casos novos de hanseníase fornecidos pelo DATASUS, selecionando-se as opções "Epidemiológicas e morbidade", "Casos de Hanseníase", "Hanseníase", "Brasil por região/unidade federativa" e "2015", "2016", "2017" e "2018". Resultados: Os dados do Brasil indicam que a região Centro-Oeste possui a maior taxa de detecção de casos

\footnotetext{
${ }^{1}$ Acadêmica de Medicina da Universidade Federal do Amapá.

${ }^{2}$ Acadêmica de Medicina da Universidade Federal do Amapá.

${ }^{3}$ Doutorado em Doenças Tropicais. Mestrado em Doenças Tropicais. Especialização em Especialização em Microbiologia. Graduação em Biomedicina.
} 
novos do Brasil $(0,135)$, seguida pelo Norte $(0,115)$ e as menores taxas estão no Sul e Sudeste $(0,011$ e 0,017, respectivamente), para cada 100 habitantes. Além disso, observa-se redução dos números de casos novos no decorrer dos anos no Nordeste, Sudeste e Sul, enquanto no Norte e Centro-Oeste foi verificado aumento destes. Dentre os Estados, Roraima apresentou o menor número de casos novos (394) no período avaliado, enquanto o Mato Grosso teve o maior número (13.195). Conclusões: A heterogeneidade na detecção de casos de hanseníase entre os Estados é elevada, podendo estar relacionada à capacidade diagnóstica dos Municípios, o acesso aos serviços de saúde, as diferenças estruturais e as especificidades das regiões, como áreas de difícil acesso e de grandes extensões territoriais. Diante disso, percebe-se que pode haver uma subnotificação entre os Estados, justificando a necessidade de promover capacitação profissional para um diagnóstico precoce, reforço das ações de vigilância e da alimentação dos sistemas de notificação e maior integração dos níveis de atenção de saúde.

Palavras-chave: Hanseníase, epidemiologia, notificação de doenças, vigilância epidemiológica, Políticas públicas.

\section{INTRODUÇÃO}

Hanseníase é uma doença infecto-contagiosa causada pelo Mycobacterium leprae ou bacilo de Hansen. O contágio ocorre através de uma pessoa doente não tratada, portadora do bacilo, que o elimina para o meio exterior, podendo contaminar pessoas susceptíveis, principalmente pelas vias aéreas. Os principais sinais e sintomas da doença são lesões na pele e nos nervos periféricos. O diagnóstico clínico é realizado através do exame físico e de uma avaliação dermatoneurológica, sendo que o tratamento compreende antibioticoterapia específica e o acompanhamento do paciente e seus contatos diretos (OLIVEIRA, DINIZ, 2016).

A hanseníase é considerada um agravo de notificação compulsória, de acordo com a portaria 204/2016, e, portanto, deve ser notificada à Secretaria de Saúde do município de atendimento do paciente em caso de suspeita ou confirmação do diagnóstico. Essa notificação é feita através do preenchimento da Ficha de Notificação/Investigação de 
Hanseníase, disponibilizada pelo Ministério da Saúde e computada pelo Sistema de Informação de Agravos de Notificação (SINAN) (BRASIL, 2016).

Desde 1991, estratégias são criadas pela Organização Mundial de Saúde (OMS) a fim de reduzir a prevalência da hanseníase, principalmente em países endêmicos e em desenvolvimento, objetivando que essa doença não seja mais um problema de saúde pública, conceituado como prevalência maior que 1/10.000 habitantes (UWIMANA et al., 2017).

Em 2016, a OMS propôs a Estratégia Global para Hanseníase 2016-2020 que visa a detecção precoce da doença e o tratamento imediato para evitar a incapacidade e reduzir a transmissão da infecção. Adicionalmente, a nível nacional, o Ministério da Saúde criou o Programa Nacional de Eliminação da Hanseníase, o qual visa desenvolver um conjunto de ações para orientar os diferentes níveis de complexidade dos serviços de saúde, fortalecendo as ações de vigilância epidemiológica da hanseníase com a criação dos sistemas de notificação e desenvolvendo ações de promoção da saúde com base na educação (BRASIL, 2018; WHO, 2016).

Segundo a OMS, em 2017, foram registrados 211.009 novos casos no mundo. Neste mesmo ano, no Brasil, foram registrados 26.689 casos novos de Hanseníase, sendo que 5.092 estão na região Norte (DATASUS, 2017). Nesse contexto, o Programa Mundial de Hanseníase (PMH) da OMS publicou o Guia de Monitoramento e Avaliação- Estratégia Global de Hanseníase 2016-2020 que visa orientar os programas sobre métodos de monitoramento de progresso e identificação de problemas (WHO, 2016).

Conhecer o perfil epidemiológico da doença faz-se necessário para avaliar a efetividade das políticas de erradicação 


\section{OBJETIVOS}

Caracterizar o panorama epidemiológico da Hanseníase entre as macrorregiões brasileiras, relatando o quantitativo de casos novos notificados e a taxa de detecção de casos novos por 100 habitantes, no período de 2015 a 2018.

\section{MÉTODOS}

Foi realizada pesquisa epidemiológica analítica, retrospectiva, transversal que utilizou dados do Departamento de Informática do Sistema Único de Saúde (DATASUS) no período de 2015 a 2018, de todas as unidades federativas do Brasil. Esse banco de dados é abastecido com casos de hanseníase notificados por meio da Ficha Individual de Notificação/ Investigação de Hanseníase, arquivada no Sistema de Informação de Agravos de Notificação (SINAN).

As informações foram obtidas no endereço eletrônico http://datasus.saude.gov.br/, selecionando-se as opções "acesso a informação", "informações em saúde (TABNET)", "Epidemiológicas e morbidade", "Casos de Hanseníase- desde 2001 (SINAN)", "Hanseníase- desde 2001", "Brasil por região/unidade federativa". Em linha, selecionou-se "Unidade da Federação"; em coluna selecionou-se "não ativa", em conteúdo selecionou-se "casos novos" e em período selecionou-se "2015", em seguida, "2016", "2017" e "2018".

Os dados obtidos foram tabelados na planilha eletrônica do Microsoft Excel, para confecção de gráficos e tabelas. Foi realizado o teste estatístico Qui-Quadrado, com analise de partição, através do programa BIOESTAT 5.3 (Ayres et al., 2007), sendo o nível de significância considerado $p<0,05$.

\section{RESULTADOS}

Dentre as macroregiões brasileiras, as que apresentaram maiores taxas de detecção de casos novos, considerando o tamanho populacional, foram as regiões Centrooeste, Norte e Nordeste, respectivamente. As regiões Sul e Sudeste apresentam as 
menores taxas de detecção de casos novos do país (0,01\% e 0,02\%) (gráfico 1). Essa diferença foi estatisticamente significativa, tendo $p<0.0001$.

Gráfico 1 Quantitativo de casos de hanseníase por 100 habitantes, notificados em 2015 a 2018 entre as regiões brasileiras.

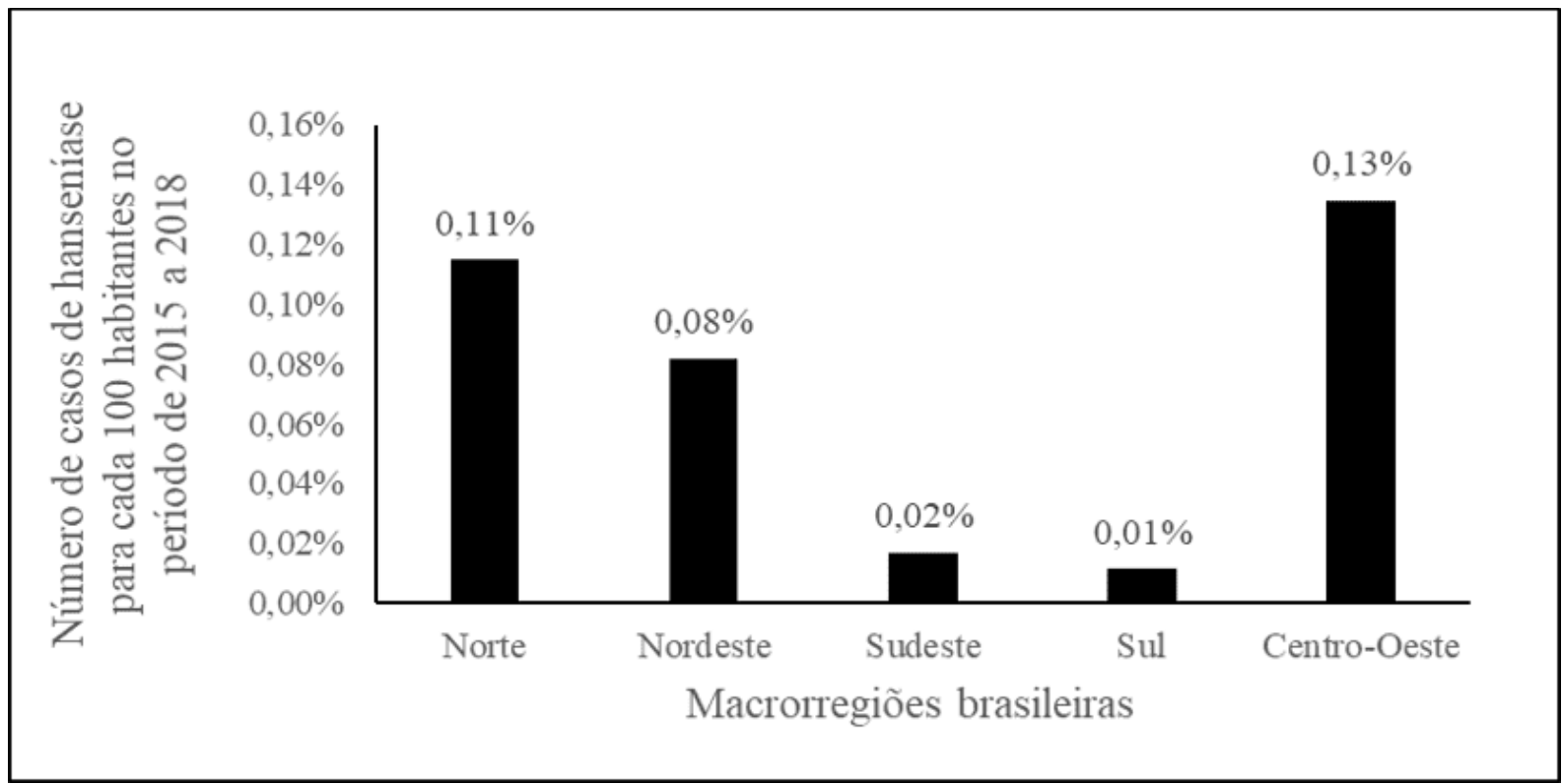

Graph 1 Quantitative leprosy's cases per 100 habitants, reported in 2015 to 2018 among brazilian regions.

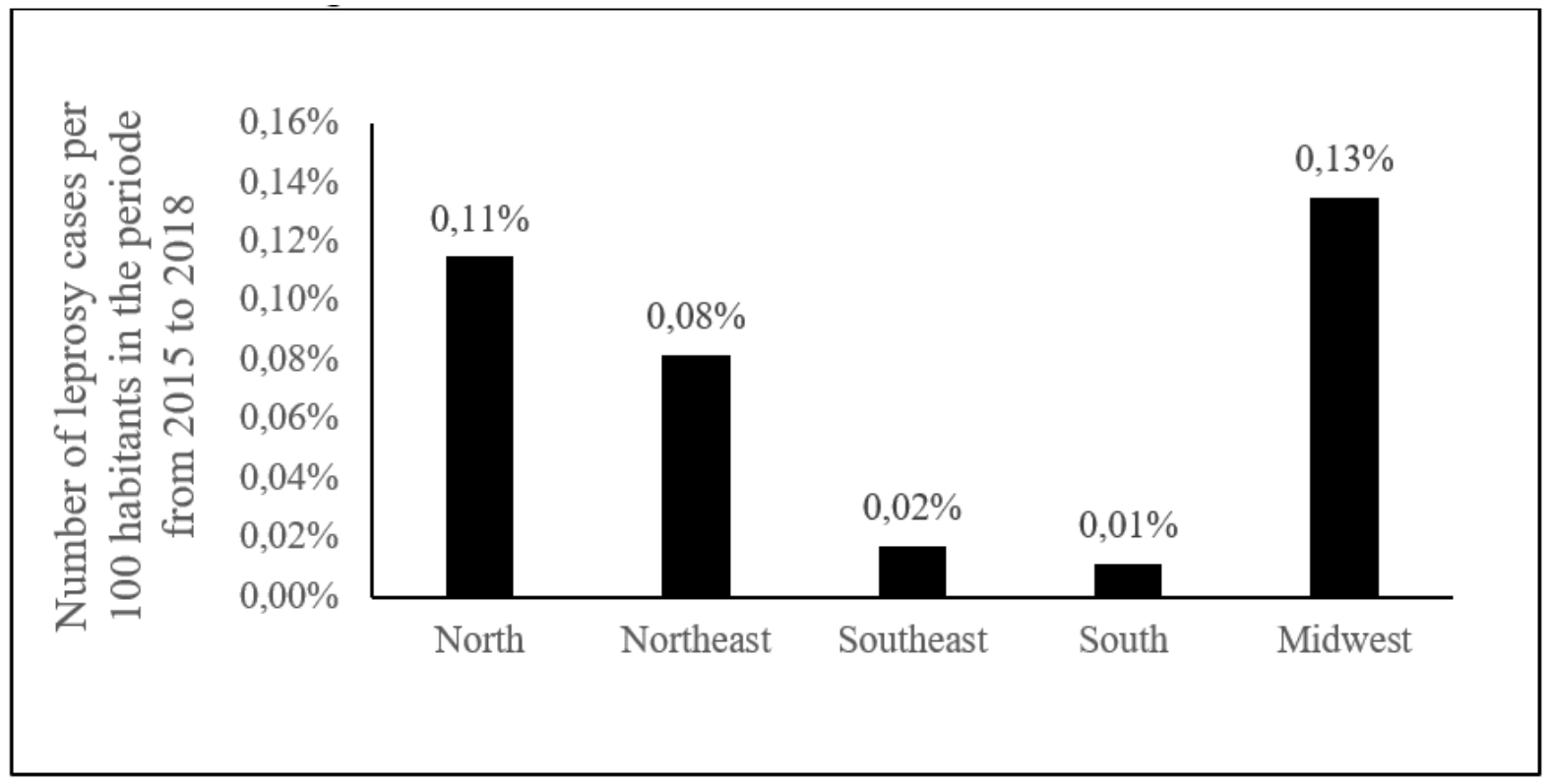


Avaliando o quantitativo de casos no decorrer do período estudado, observa-se uma tendência de redução nos números detectados nas regiões Nordeste, Sudeste e Sul, enquanto as regiões Norte e Centro-Oeste verificou-se discreto aumento (gráfico 2).

Gráfico 2 Quantitativo de casos de Hanseníase notificados a cada ano nas regiões do Brasil, de 2015 a 2018.

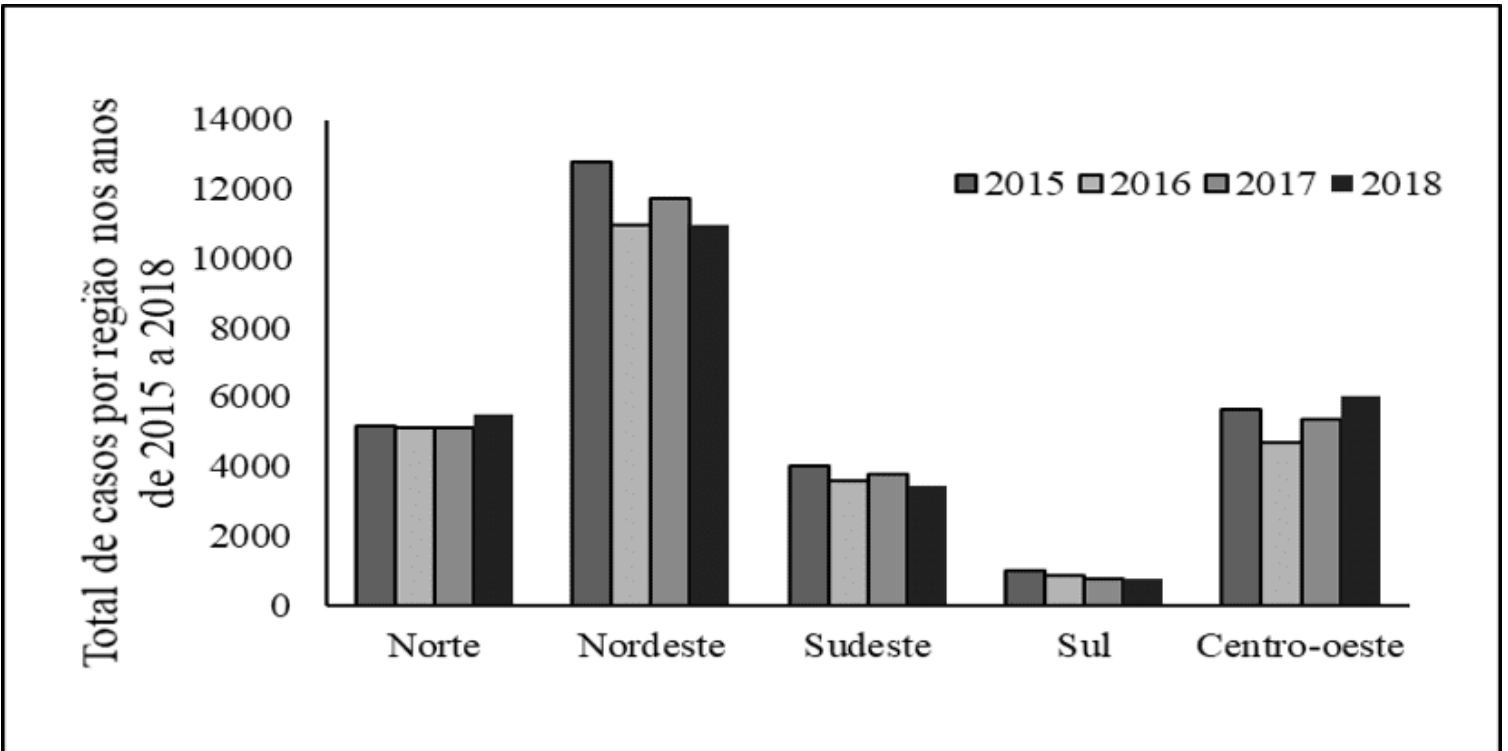

Graph 2 Quantitative leprosy's cases reported each year in the regions of Brazil, from 2015 to 2018

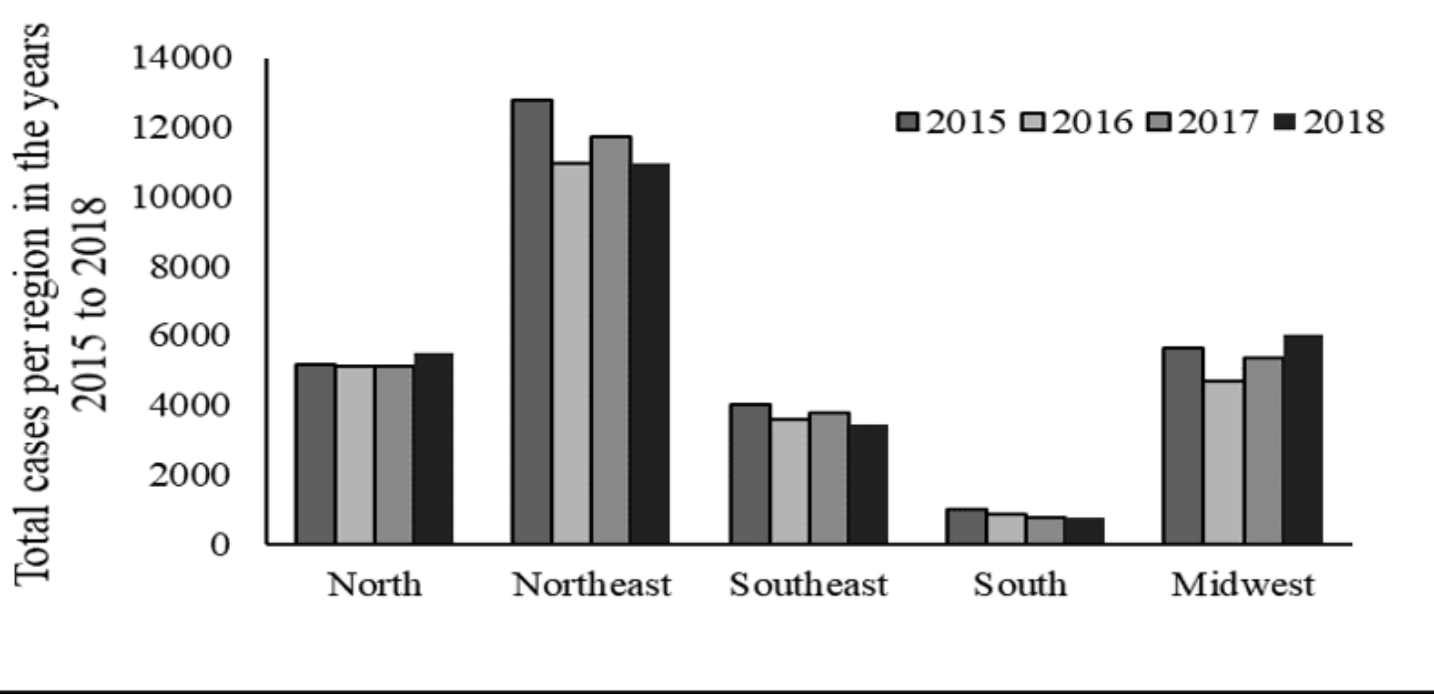


Dentre os Estados, Roraima apresentou o menor número de novos casos, seguido do Estado do Amapá, mas as menores taxas de detecção encontram-se nos Estados do Sul, como Rio Grande do Sul e Santa Catarina. Em contrapartida, o maior número de casos novos foi encontrado no Estado do Mato Grosso, seguido por Maranhão e Pará e as maiores taxas de detecção em Tocantins. Avaliando apenas a região Norte, os dados mostram maior número de casos novos no Pará e maior taxa de detecção em Tocantins, de 0,32\%. O Estado com menor número de casos novos é Roraima e com menor taxa de detecção é Amazonas, com 0,04\%. A distribuição dos casos encontrase descritas na tabela1. 
Tabela 1 Número total de Casos novos de Hanseníase e número total de casos para cada 100 habitantes no Brasil, Regiões e Estados, de 2015 a 2018.

\begin{tabular}{|c|c|c|c|}
\hline & $\begin{array}{c}\text { Número de casos de } \\
\text { Hanseniase nos anos de } \\
2015 \text { a } 2018\end{array}$ & $\begin{array}{l}\text { Número de casos para } \\
\text { cada } 100 \text { habitantes }\end{array}$ & $\mathrm{p}$ \\
\hline Região Norte & 20866 & $0,115 \%$ & $<0.0001$ \\
\hline Rondônia & 2259 & $0,1285 \%$ & \\
\hline Acre & 498 & $0,0573 \%$ & \\
\hline Amazonas & 1832 & $0,0449 \%$ & \\
\hline Roraima & 394 & $0,0683 \%$ & \\
\hline Pará & 10343 & $0,1215 \%$ & \\
\hline Amapá & 418 & $0,0504 \%$ & \\
\hline Tocantins & 5122 & $0,3293 \%$ & \\
\hline Região Nordeste & 46428 & $0,0820 \%$ & $<0.0001$ \\
\hline Maranhão & 12893 & $0,1833 \%$ & \\
\hline Piaui & 3954 & $0,1211 \%$ & \\
\hline Ceará & 6471 & $0,0713 \%$ & \\
\hline Rio Grande do Norte & 970 & $0,0279 \%$ & \\
\hline Paraiba & 1854 & $0,0464 \%$ & \\
\hline Pernanbuco & 8829 & $0,0930 \%$ & \\
\hline Alagoas & 1269 & $0,0382 \%$ & \\
\hline Sergipe & 1339 & $0,0588 \%$ & \\
\hline Bahia & 8849 & $0,0597 \%$ & \\
\hline Região Sudeste & 14791 & $0,0170 \%$ & $<0.0001$ \\
\hline Minas Gerais & 4340 & $0,0206 \%$ & \\
\hline Espirito Santo & 1987 & $0,0500 \%$ & \\
\hline Rio de Janeiro & 3579 & $0,0209 \%$ & \\
\hline São Paulo & 4885 & $0,0107 \%$ & \\
\hline Região Sul & 3375 & $0,0110 \%$ & $<0.0001$ \\
\hline Paraná & 2383 & $0,0210 \%$ & \\
\hline Santa Catarina & 544 & $0,0077 \%$ & \\
\hline Rio Grande do Sul & 448 & $0,0040 \%$ & \\
\hline Região Centro-Oeste & 21666 & $0,1350 \%$ & $<0.0001$ \\
\hline Mato Grosso do Sul & 1829 & $0,0666 \%$ & \\
\hline Mato Grosso & 13195 & $0,3834 \%$ & \\
\hline Goiás & 5915 & $0,0855 \%$ & \\
\hline Distrito Federal & 727 & $0,0244 \%$ & \\
\hline Bra sil & 107126 & $0,0514 \%$ & $<0.0001$ \\
\hline
\end{tabular}


Table 1 Total number of leprosy's new cases and total number of cases for every 100 habitant in Brazil, Regions and States, from 2015 to 2018

Number ofleprosy's cases in Number of cases per the years of 2015 to 2018 $\mathrm{p}$

\begin{tabular}{lccc}
\hline Região Norte & 20866 & $0.115 \%$ & $<0.0001$ \\
\hline Rondônia & 2259 & $0.1285 \%$ & \\
Acre & 498 & $0.0573 \%$ & \\
Amazonas & 1832 & $0.0449 \%$ & \\
Ror aima & 394 & $0.0683 \%$ & \\
Pará & 10343 & $0.1215 \%$ & \\
Amapá & 418 & $0.0504 \%$ & \\
Tocantins & 5122 & $0.3293 \%$ & \\
\hline Região Nordeste & 46428 & $0.0820 \%$ & $<0.0001$ \\
\hline Maranhão & 12893 & $0.1833 \%$ & \\
Piaui & 3954 & $0.1211 \%$ & \\
Ceará & 6471 & $0.0713 \%$ & \\
Rio Grande do Norte & 970 & $0.0279 \%$ & \\
Paraiba & 1854 & $0.0464 \%$ & \\
Pernanbuco & 8829 & $0.0930 \%$ & \\
Alagoas & 1269 & $0.0382 \%$ & \\
Sergipe & 1339 & $0.0588 \%$ & \\
Bahia & 8849 & $0.0597 \%$ & \\
\hline Região Sudeste & 14791 & $0.0170 \%$ & $<0.0001$ \\
\hline Minas Gerais & 4340 & $0.0206 \%$ & \\
Espirito Santo & 1987 & $0.0500 \%$ & \\
Rio de Janeiro & 3579 & $0.0209 \%$ & \\
São Paulo & 4885 & $0.0107 \%$ & \\
\hline Região Sul & 3375 & $0.0110 \%$ & \\
\hline Paraná & 2383 & $0.0210 \%$ & \\
Santa Catarina & 544 & $0.0077 \%$ & \\
Rio Grande do Sul & 448 & $0.0040 \%$ & \\
\hline Região Centro-Oeste & 107126 & $0.1350 \%$ & \\
\hline Mato Grosso do Sul & & $0.0666 \%$ & \\
Mato Grosso & 13195 & $0.3834 \%$ & \\
Goiás & 5915 & $0.0855 \%$ & \\
Distrito Federal & & $0.0244 \%$ & \\
\hline Brasil & & & \\
\hline
\end{tabular}




\section{DISCUSSÃO}

As regiões Centro-oeste, Norte e Nordeste apresentaram maior registro de casos, sendo consideradas hiperendêmicas para a doença. Esse achado também foi descrito por Monteiro et al. (2017), que o relaciona com o fato de essas regiões serem consideradas as mais pobres do país, com grande número de habitantes em situação socioeconômica desfavorável. Ribeiro, Silva e Oliveira (2018), que também encontraram padrão semelhante, comparam essas regiões brasileiras com os países mais podres do Hemisfério Sul, em relação a endemia para doenças infectocontagiosas e alta morbimortalidade.

As regiões Sul e Sudeste tiveram as menores taxas de detecção no período de 2015 a 2018, com destaque para Rio Grande do Sul e Santa Catarina, que tiveram no presente estudo as menores taxas de detecção de hanseníase, atingindo a meta para eliminação da hanseníase como problema de saúde pública proposta pela OMS em 1991 (menos de 1 caso para 10.000 habitantes). Esse fato também foi apresentado por Segurado, Cassenote e Luna (2016), que apresenta o diagnóstico e tratamento precoces como principal causa deste achado.

No presente estudo verificou-se redução no número de casos detectados nas regiões Nordeste, Sudeste e Sul, enquanto as regiões Norte e Centro-Oeste apresentaram ligeiro aumento no período estudado. Ribeiro, Silva e Oliveira (2018) também observaram discrepância entre as tendências dos indicadores de hanseníase das regiões, evidenciando algo semelhante a países mais pobres do hemisfério Sul nas regiões Norte e Centro-Oeste e padrões comparáveis aos de países desenvolvidos nas regiões Sul e Sudeste. A grande extensão territorial brasileira e as desigualdades socioeconômicas são citadas como principal motivo para esta discrepância além que colaborarem para a dificuldade da manutenção da tendência de eliminação da Hanseníase em nível nacional.

Em relação a análise dos Estados brasileiros, o maior número de casos foi encontrado no Mato Grosso, seguido por Maranhão e Pará, enquanto a maior taxa de detecção foi notificada em Tocantins. Segurado et al. (2016), que obteve resultado semelhante, 
apresentou esses Estados como, além de detentores de prevalências elevadas da doença, locais de reunião de alguns dos "10 clusters" de municípios no Brasil, que concentram metade dos casos de Hanseníase do país. Esse achado também foi relatado por Freitas, Duarte e Garcia (2017), que sugeriram que isso ocorre por três mecanismos: vulnerabilidade social destas áreas, má qualidade da atenção em saúde e má qualidade da informação em saúde como fator de distorção dos dados analisados.

O presente estudo evidenciou também heterogeneidade entre os Estados de uma mesma região na detecção de casos de hanseníase (Tabela 1), como por exemplo, no caso da região Norte, os Estados de Roraima e do Amapá, que apresentaram os menores números de casos do Brasil, aproximando-se da meta, ainda que façam parte de uma região hiperendêmica para a Hanseníase. Freitas, Duarte e Garcia (2017) enfatizam como principal limitação do uso de dados da vigilância epidemiológica a subnotificação de casos, explicada principalmente pela presença de casos oligossintomáticos, precariedade dos serviços de vigilância e diagnostico, além de áreas de difícil acesso geográfico.

A Hanseníase faz parte do grupo de Doenças Tropicais Negligenciadas, designado assim pela OMS, por acometerem principalmente as camadas mais pobres da população localizada nas regiões topicais. Essas doenças possuem precária atenção da indústria farmacêutica e dos representantes políticos nacionais. Isso acentua a dificuldade diagnostica, que conta com métodos laboratoriais pouco sensíveis, levando a um diagnóstico predominantemente clinico, o que normalmente atrasa o início do tratamento, aumentando o período de transmissão da doença (SEGURADO, CASSENOTE, LUNA, 2016). Além disso, o longo período necessário para o tratamento, o perfil de efeitos colaterais e o precário acompanhamento do tratamento pelos profissionais de saúde foram descritos por Souza et al. (2018) como principais causas associadas ao abandono e baixa adesão a poliquimioterapia.

Para aumentar a identificação dos casos de hanseníase no país, foi criado o Programa da Hanseníase no Brasil que integrado ao SINAN visa garantir a padronização da notificação com maior agilidade da operação do sistema, a partir da informatização 
dos dados. Todavia, um entrave à real eficácia desse projeto ainda se constitui do não preenchimento completo e correto dos instrumentos de notificação, lentidão dos processos de informatização dos dados, além da precária busca ativa de casos. Com isso, torna-se necessário avaliações sistemáticas da qualidade dos dados notificados e capacitação dos profissionais sobre o preenchimento e captação dos dados, além da importância destes para a elaboração de políticas públicas eficazes e respaldo cientifico para pesquisas na área (AGUIAR, 2015).

\section{CONSIDERAÇÕES FINAIS}

As diferenças nos índices de hanseníase no Brasil parecem possuir raízes multifatoriais, pautadas principalmente na condição socioeconômica das regiões, sendo os maiores números encontrados em regiões menos desenvolvidas, como Norte, Nordeste e Centro-Oeste. A má qualidade da atenção em saúde e a grande extensão territorial brasileira também podem ser motivos para essa discrepância e para a manutenção do padrão infectocontagioso das morbidades dessas regiões.

Os resultados demonstram que existe o desafio de tornar a notificação elevada e homogênea nas diferentes unidades federativas do país. Isso se deve principalmente às diferenças estruturais e especificidades das regiões, como grandes extensões territoriais, áreas de difícil acesso geográfico, diagnostico tardio da doença, e precariedade dos serviços de vigilância.

A capacitação de profissionais para um diagnóstico precoce e mais eficaz de novos casos, reforço das ações de vigilância e alimentação dos sistemas de notificação da hanseníase, além de maior integração de todos os níveis de atenção de saúde são necessários e poderiam contribuir para obtenção de dados mais compatíveis com a realidade, o que refletiria em um reforço das ações de saúde criadas pelo Ministério da Saúde e pela Vigilância Epidemiológica com o reconhecimento da magnitude da incidência da doença na região. 


\section{REFERÊNCIAS}

AGUIAR, Yatamiris Pâmela da Silva. Qualidade dos registros de Hanseníase no sistema de informação de agravos de notificação em Teresina, Piauí, 2012. 2015. 82 f. Dissertação (Mestrado) - Curso de Epidemiologia em Saúde Pública, Escola Nacional de Saúde Pública Sergio Arouca, Rio de Janeiro, 2015.

AYRES, Manoel; AYRES JR, Manoel; AYRES, Daniel Lima; SANTOS,Alex de Assis. BioEstat 5.3: aplicações estatísticas nas áreas das ciências biológicas e médicas. Belém: IDSM, 2007. 364p.

BRASIL. Ministério da Saúde. Boletim Epidemiológico Hanseníase. Secretaria de Vigilância em Saúde, v.49, n.4, p. 1-12, 2018.

BRASIL. Ministério da Saúde. Portaria №. 204, de 17 de fevereiro de 2016. Define a Lista Nacional de Notificação Compulsória de doenças, agravos e eventos de saúde pública nos serviços de saúde públicos e privados em todo o território nacional, nos termos do anexo, e dá outras providências. Diário Oficial da União 18 fev 2016; Seção 1.

DATASUS. Departamento de Informática do SUS, 2017. Acompanhamento da Hanseníase- Brasil 2014. [cited 2017 dez 5] Available from http://tabnet.datasus.gov.br/cgi/tabcgi.exe?sinannet/hanseniase/cnv/hanswuf.def

FREITAS, Lúcia Rolim Santana de; DUARTE, Elisabeth Carmen; GARCIA, Leila Posenato. Análise da situação epidemiológica da hanseníase em uma área endêmica no Brasil: distribuição espacial dos períodos 2001 - 2003 e 2010 - 2012. Revista Brasileira de Epidemiologia, Brasília, v. 20, n. 4, p.702-713, dez. 2017. FapUNIFESP (SciELO). http://dx.doi.org/10.1590/1980-5497201700040012.

MONTEIRO, Lorena Dias; MOTA, Rosa Maria Salani; MELO, Francisco Rogerlândio Martins; ALENCAR, Carlos Henrique; HEUKELBACH, Jorg. Social determinants of leprosy in a hyperendemic State in North Brazil. Revista de Saúde Pública, [s.I.], v. 
51, p.1-1, 20 jul. 2017. FapUNIFESP (SciELO). http://dx.doi.org/10.1590/s15188787.2017051006655 .

OLIVEIRA, Marcela Bahia Barretto de; DINIZ, Lucia Martins. Leprosy among children under 15 years of age: literature review. Anais Brasileiros de Dermatologia, [s.I.], v. 91, n. 2, p.196-203, abr. 2016. FapUNIFESP (SciELO). http://dx.doi.org/10.1590/abd1806-4841.20163661.

RIBEIRO, Mara Dayanne; SILVA, Jefferson Carlos; OLIVEIRA, Sabrynna. Estudo epidemiológico da hanseníase no Brasil: reflexão sobre as metas de eliminação. Revista Panamericana de Salud Pública, [s.l.], p.1-7, 2018. Pan American Health Organization. http://dx.doi.org/10.26633/rpsp.2018.42.

SEGURADO, Aluisio Cotrim; CASSENOTE, Alex Jones; LUNA, Expedito de Albuquerque. Saúde nas metrópoles - Doenças infecciosas. Estudos Avançados, São Paulo, v. 30, n. 86, p.29-49, abr. 2016. FapUNIFESP (SciELO). http://dx.doi.org/10.1590/s0103-40142016.00100003.

SOUZA, Eliana Amorim de; Boigny, Reagan Nzundu; Ferreira, Anderson Fuertes; Alencar, Carlos Henrique; Oliveira, Maria Leide W.; Ramos Jr., Alberto Novaes. Vulnerabilidade programática no controle da hanseníase: padrões na perspectiva de gênero no Estado da Bahia, Brasil. Cadernos de Saúde Pública, [s.I.], v. 34, n. 1, p.1-14, 5 fev. 2018. FapUNIFESP (SciELO). http://dx.doi.org/10.1590/0102$311 \times 00196216$.

UWIMANA, Innocent; BIZIMUNGU, Nestor; INGABIRE, Fabrice; MUKAMUKWIYE, Elyse; SHRANGABO, Odette; NGABONZIZA, Semuto C.; KAMANZI, Elaine. Trends in leprosy case detection in Rwanda, 1995-2011: analysis of 17 years of laboratory data. African Journal Of Laboratory Medicine, [s.I.], v. 6, n. 1, p.426-426, 24 fev. 2017. AOSIS. http://dx.doi.org/10.4102/ajlm.v6i1.426.

WHO. World Health Organization 2016. Global Leprosy Strategy: Accelerating towards a leprosy-free world. [cited 2017 dez 6] Available from http://apps.who.int/iris/bitstream/10665/208824/14/9789290225096_en.pdf?ua=1 
Enviado: Janeiro, 2020.

Aprovado: Março, 2020. 